\title{
Evolution of interacting binaries with a B type primary at birth
}

\author{
W. Van Rensbergen, C. De Loore, and K. Jansen
}

Astrophysical Institute, Vrije Universiteit Brussel, Pleinlaan 2, 1050 Brussels, Belgium

e-mail: wvanrens@vub.ac.be; cdeloore@pandora.be; kijansen@vub.ac.be

Received 31 May 2005 / Accepted 13 September 2005

\section{ABSTRACT}

We revisited the analytical expression for the mass ratio distribution for non-evolved binaries with a B type primary. Selection effects governing the observations were taken into account in order to compare theory with observations. Theory was optimized so as to fit best with the observed $q$-distribution of SB1s and SB2s. The accuracy of this theoretical mass ratio distribution function is severely hindered by the uncertainties on the observations. We present a library of evolutionary computations for binaries with a B type primary at birth. Some liberal computations including loss of mass and angular momentum during binary evolution are added to an extensive grid of conservative calculations. Our computations are compared statistically to the observed distributions of orbital periods and mass ratios of Algols. Conservative Roche Lobe Over Flow (RLOF) reproduces the observed distribution of orbital periods but fails to explain the observed mass ratios in the range $q \in[0.4-1]$. In order to obtain a better fit the binaries have to lose a significant amount of matter, without losing much angular momentum.

Key words. stars: binaries: close - stars: evolution - stars: fundamental parameters - stars: mass-loss stars: luminosity function, mass function - stars: statistics

\section{Introduction}

The mass ratio distribution of binary stars was treated extensively by Hogeveen (1991). He showed how an observed $q$-distribution can be transformed into a real one. We revisited the Hogeveen method and applied it onto the population of nonevolved spectroscopic binaries (SBs) with a B type primary. We used the new $q$-distribution to compute the evolution of binaries with a B type primary at birth beyond the Algol stage. Eggleton (2000) introduced the word "liberal" to distinguish binary evolution with mass and angular momentum loss from the conservative case where no mass and consequently no angular momentum leave the system. Refsdal et al. (1974) showed that the binary AS Eri is the result of liberal binary evolution; the amounts of mass and angular momentum lost by the system however are uncertain. Sarna (1993) showed that during RLOF only $60 \%$ of the mass lost by the loser of $\beta$ Per was captured by the gainer and that $30 \%$ of the initial angular momentum was lost. Hence it is clear that a liberal treatment is important for binary evolution calculations. However the amount of mass as well as angular momentum that has to be removed from interacting systems is far from obvious. Considering the examples mentioned above it may be expected that the match between observations and conservative calculations will not be satisfactory. Therefore we add liberal calculations to our conservative library. The detailed evolutionary tracks can be found in http: //www.vub.ac.be/astrofys/

With the Brussels simultaneous evolution code (see e.g. "The Brightest Binaries", Vanbeveren et al. 1998) we calculated a representative grid of the conservative evolution of binaries with a B type primary at birth. Application of the criterion of Peters (2001) allows then to determine for each of the evolutionary sequences the beginning and end of various Algol stages. This criterion states that in the semi-detached system:

- The less massive star fills its Roche lobe (RL).

- The most massive star does not fill its RL and is still on the main sequence.

- The less massive star is the cooler, the fainter and the larger.

The grid allows us to determine an expected distribution of orbital periods and mass ratios for Algols. This can then be compared to the observations.

\section{Unevolved binaries}

\subsection{Observed mass ratios of non-evolved binaries}

From the 9th Catalogue of Spectroscopic Binaries (Pourbaix et al. 2004) which we used in its on-line version (Pourbaix 2005), we selected 142 SBs with a non-evolved early B type [B0-B3] and 146 with a non-evolved late B type primary [B4-B9.5] as follows: starting with a set of binaries with a B type primary we kept only those with primaries of luminosity class III-V. We disregarded all Algols and systems with suffixes $n$ and $e$. We excluded those binaries which were classified as semi-detached or contact by Brancewicz et al. (1996) or quoted as showing emission lines in the SIMBAD archive (http://simbad.u-strasbg.fr/Simbad/). 
Table 1. HD or SB9 numbers of the 288 SBs used in this analysis.

\begin{tabular}{|c|c|}
\hline SB type & HD number or SB9 number if no HD number is available \\
\hline 46 Early B SB2s & $\begin{array}{c}\text { 3950, 23180, 23625, 24760, 29376, 29763, 35715, 36695, 37021, 37756, 39698, 52942, } 65818 \\
\text { 75759, 77464, 86118, 90707, 104337, 115937, 116658, 121263, 136504, 139365, 143018 } \\
\text { 144217, 147971, 151890, 156633, 161783, 164852, 175544, 176853, 177284, 177624, } 185507 \\
191201,191567,193536,193611,197770,208947,217312,218440,227696,305543, \text { SB9 number: } 1768\end{array}$ \\
\hline 96 Early B SB1s & $\begin{array}{c}3264,3901,16908,23466,24190,25799,30836,31237,32990,35039 \\
35588,36485,36695,36822,36954,37017,37202,37438,40005,41753,42560,42933,44402,46792 \\
56310,59543,64503,65041,68243,69144,74560,75655,75821,79351,81188,87059,92287,93030 \\
104841,108248,113791,120307,134687,138690,142096,142669,142883,143275,145482,145502 \\
147165,149834,149881,160762,175426,176819,178329,180163,188753,189103,191473,193516 \\
193924,198784,200776,205021,206672,207330,209961,213420,214240,214652,216711,216916 \\
\text { 217224, 217463, 217919, 217979, 218342, 218407, 221253, 235807, 240068 } \\
\text { SB9 numbers: } 319,320,928,1344,1367,1374,1414,1777,1778,1779,1780,1791,1951 \\
\end{array}$ \\
\hline 41 Late B SB2s & $\begin{array}{c}2019,3369,4727,6118,6882,12534,21278,22203,25833,27376,28475,32419,32964,34364,39357 \\
41040,44172,44701,75920,96314,112014,123515,136175,139892,140008,140873,147683,156247,162028 \\
162724,165814,178322,191110,191692,196628,199081,208095,212120,216494,224113,240058\end{array}$ \\
\hline 105 Late B SB1s & $\begin{array}{c}1976,2054,3322,4382,4775,5408,7374,11291,11529,16219,16907,17081,17543,20315 \\
21279,23850,23964,24587,24769,25487,27295,30211,34759,34762,35079,36881,37000,41040,58661 \\
61926,62737,63251,68351,68520,72208,74146,75446,75642,76805,77350,78316,86360,86619,87191 \\
87751,92190,93191,93549,95878,96307,101897,102465,114911,120710,120955,134759,137432,137569 \\
139160,139892,141556,142315,145389,159082,161165,161480,161573,161660,161701,162515,162630 \\
162656,162679,162780,169978,170200,170465,173886,174403,174714,174933,176318,177863,178125 \\
180553,180554,183056,183794,185936,186688,190229,192276,193495,193964,195986,197226,199892 \\
201359,207857,211838,219749,220318, \text { SB9 numbers: } 1191,1288,1289\end{array}$ \\
\hline
\end{tabular}

Table 1 lists the binaries that have been used in this analysis.

The mass ratio for SB2s was determined from observations for the primary (subscript 1) and the companion (subscript 2):

$q=\frac{M_{2}}{M_{1}}=\frac{K_{1}}{K_{2}}=\frac{a_{1}}{a_{2}}$

$q$ is the mass ratio, $M$ the mass, $K$ the maximum radial orbital velocity and $a$ the semi-major axis of the orbital ellipse. The results are included in Table 2 . The mass ratio for SB1s was determined from the mass function $f(m)$ :

$f(m)=\frac{M_{2}^{3} \sin ^{3} i}{\left(M_{1}+M_{2}\right)^{2}}=\left(1.0385 \times 10^{-7}\right) K_{1}^{3}\left(1-e^{2}\right)^{\frac{3}{2}} P M_{\odot}$

with $K$ in $\mathrm{km} \mathrm{s}^{-1}$ and the orbital period $\mathrm{P}$ in days. In order to derive $q=\frac{M_{2}}{M_{1}}$ from $f(m)$ the mass of the primary was taken from Vanbeveren et al. (1998) for early B primaries. For late B primaries we used the evolutionary tracks from Schaller et al. (1992). In that case, the mass of a star on a track in the middle between the ZAMS and the TAMS has been taken as representative for a particular spectral type. Abt \& Levy (1985) proposed to evaluate the mass ratio using relation (2) sampled at $i=15^{\circ}, 45^{\circ}$ and $75^{\circ}$, representing respectively the intervals $\left[0^{\circ}-30^{\circ}\right],\left[30^{\circ}-60^{\circ}\right]$ and $\left[60^{\circ}-90^{\circ}\right]$. Assuming a random distribution of the inclination angle $i$, one writes the fractional number of systems in the range $[i, i+\mathrm{d} i]$ as $\mathrm{d} f=\sin i \mathrm{~d} i$. The probability of the inclination angle occurring between $\alpha$ and $\beta$ is thus $f[\alpha-\beta]=\cos \alpha-\cos \beta$. This determines $f\left(15^{\circ}\right)=$ $0.134, f\left(45^{\circ}\right)=0.366$ and $f\left(75^{\circ}\right)=0.5$. A more refined method of Van Rensbergen (2001), evaluates relation (2) in the range $\left[i_{\min }-90^{\circ}\right]$. The value of $i_{\min }$ is the smallest value of the inclination for which expression (2) is meaningful. Figure 1 considers HD 37021: a SB2 with $q=0.388$ and a B3V-primary. The range $\left[i_{\min }-90^{\circ}\right]$ determines for a range of possible mass ratios between $q_{\min }\left(i=90^{\circ}\right)$ and $q_{\max }\left(i=i_{\min }\right)$.

It has been shown by Van Rensbergen (2001) that both methods can equally well be used in a statistical analysis. The probability for a mass ratio to occur in the five $q$-bins is shown in Table 2. The mass ratio of a SB2 is taken directly from http://sb9.astro.ulb.ac.be/, whereas the distribution of the mass ratios of the SB1s has been derived with the method shown in Fig. 1. The uncertainties on these numbers included in Table 2 depend on the uncertainties quoted in SB9. Those uncertainties are discussed in Sect. 2.2.

\subsection{Modeling the uncertainties in the catalogue}

The observed mass ratios of non-evolved binaries quoted as "mean" in Table 2 are determined from the mean values of $K_{1}$, $K_{2}$ and $f(m)$ in Pourbaix (2005). We have calculated the errors on these results using two extreme assumptions on the 66 systems of the SB9 catalogue for which a standard deviation on the measurements of $K_{1}, K_{2}$ and $f(m)$ is given. We have applied these data to the entire sample of 288 systems.

- The observed distribution quoted as "low $q$ " is obtained when all the uncertainties act together to reduce the values of $q$. 
Table 2. Distributions of the mass ratio of $288 \mathrm{SBs}$ with a non-evolved B type primary, used to perform our simulations.

\begin{tabular}{cccccc}
\hline \hline q-bin & ] $0-0.2]$ & {$[0.2-0.4]$} & {$[0.4-0.6]$} & {$[0.6-0.8]$} & {$[0.8-1]$} \\
\hline 46 Early B SB2s (low $q$ ) & 0 & 5 & 10 & 22.667 & 8.333 \\
46 Early B SB2s (mean) & 0 & 5 & 10 & 15 & 16 \\
46 Early B SB2s (high $q$ ) & 0 & 1.167 & 13.833 & 11.167 & 19.833 \\
96 Early B SB1s (low $q$ ) & 42.097 & 28.270 & 14.207 & 6.748 & 4.677 \\
96 Early B SB1s (mean) & 38.736 & 30.326 & 14.975 & 7.120 & 4.843 \\
96 Early B SB1s (high $q$ ) & 33.334 & 34.364 & 15.791 & 7.500 & 5.010 \\
142 Early B SBs (low $q$ ) & 42.097 & 33.270 & 24.207 & 29.415 & 13.011 \\
142 Early B SBs (mean) & 38.736 & 35.326 & 24.975 & 22.120 & 20.843 \\
142 Early B SBs (high $q$ ) & 33.334 & 35.531 & 29.625 & 18.667 & 24.843 \\
\hline 41 Late B SB2s (low q) & 0 & 1 & 6 & 9 & 25 \\
41 Late B SB2s (mean) & 0 & 1 & 6 & 9 & 25 \\
41 Late B SB2s (high $q$ ) & 0 & 1 & 6 & 3.875 & 30.125 \\
105 Late B SB1s (low $q$ ) & 37.695 & 32.208 & 17.404 & 10.249 & 7.450 \\
105 Late B SB1s (mean) & 29.976 & 36.028 & 18.646 & 12.082 & 8.268 \\
105 Late B SB1s (mean) & 24.762 & 36.750 & 21.823 & 12.823 & 9.240 \\
146 Late B SBs (low q) & 37.695 & 33.203 & 23.404 & 19.249 & 32450 \\
146 Late B SBs (mean) & 29.976 & 37.028 & 24.646 & 21.082 & 33.268 \\
146 Late B SBs (high $q$ ) & 24.762 & 37.750 & 27.426 & 16.698 & 39.365 \\
\hline
\end{tabular}

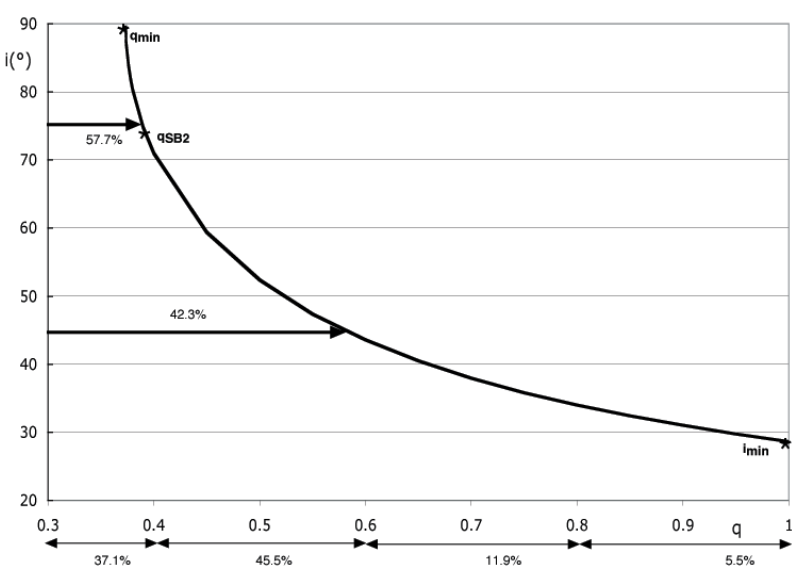

Fig. 1. Relation between $q$ and i imposed by relation (2) for a SB1. Here HD 37021 is shown. Abt \& Levy (1985) give $q=0.39$ with a probability $f(q) \approx 57.7 \%$ and $q=0.58$ with $f(q) \approx 42.3 \%$. Here we find $q \in[0.2-0.4]$ with $f(q) \approx 37.1 \%, q \in[0.4-0.6]$ with $f(q) \approx 45.5 \%$, $q \in[0.6-0.8]$ with $f(q) \approx 11.9 \%$ and $q \in[0.8-1]$ with $f(q) \approx 5.5 \%$. Since HD 37021 is a SB2, the real value of $q=0.388$ and $i=75^{\circ}$.

- The observed distribution quoted as "high q" is obtained when all the uncertainties act together to enhance the values of $q$.

It is clear that the "mean" distribution settles between these two very extreme possibilities.

\subsection{The theoretical mass ratio distribution}

Consider a non-evolved binary with total mass $M$ containing a primary with mass $M_{1}$. In our simulation we restrict $M_{1}$ to the following intervals:

- $M_{1} \in$ [7-16.7] $M_{\odot}$ for early B primaries, Vanbeveren et al. (1998).
- $M_{1} \in[2.5-7] M_{\odot}$ for late B primaries, Schaller et al. (1992).

Figure 2 shows how each binary can be located in the $\left[M_{1}-M\right]$-plane, in which straight lines defined by $M=M_{1} \times$ $(q+1)$ are loci of constant $q$. Each point in the domain is weighted with its probability of occurrence. The fraction of binaries with primary mass $\in\left[M_{1, \min }-M_{1, \max }\right]$ and $q \in[q-q+\mathrm{d} q]$ is the weighted black surface divided by the weighted grey surface of Fig. 2.

We assume that the mass of the primary follows a normalised Salpeter-like IMF (Salpeter 1955):

$\xi\left(M_{1}\right)=C \times M_{1}^{-\alpha}$ with $C=\frac{1-\alpha}{M_{1, \max }^{1-\alpha}-M_{1, \min }^{1-\alpha}}$.

Vanbeveren (1982) showed that the exponent $\alpha$ needs not be the same for primaries of binaries as for single stars. For single stars, Salpeter (1955) proposed $\alpha=2.35$. Rana (1987) argued $\alpha=1.5$ for $M>1.6 M_{\odot}$. Elmegreen (1997) finds $\alpha \in$ [2.5-3] for field stars and $\in$ [2-2.5] for stars in clusters.

The probability that this primary is bound to a secondary with mass $M_{2}=M-M_{1}$ is:

$$
\begin{array}{ll}
\xi\left(M, M_{1}\right)=f\left(M_{1}\right) \times M^{-\delta} & \text { if } M \in\left[M_{1}-2 \times M_{1}\right] \\
\xi\left(M, M_{1}\right)=0 & \text { if } M \notin\left[M_{1}-2 \times M_{1}\right] .
\end{array}
$$

The shape of the function $f\left(M_{1}\right) \propto M_{1}^{\delta-1}$ is unambiguously defined from the condition of normalisation:

$\int_{M_{1}}^{2 \times M_{1}} \xi\left(M, M_{1}\right) \mathrm{d} M=1$.

The probabilty of occurrence of a binary: i.e. the weight of each point in the grey domain of Fig. 2 is thus:

$\xi\left(M, M_{1}\right) \times \xi\left(M_{1}\right)$. 


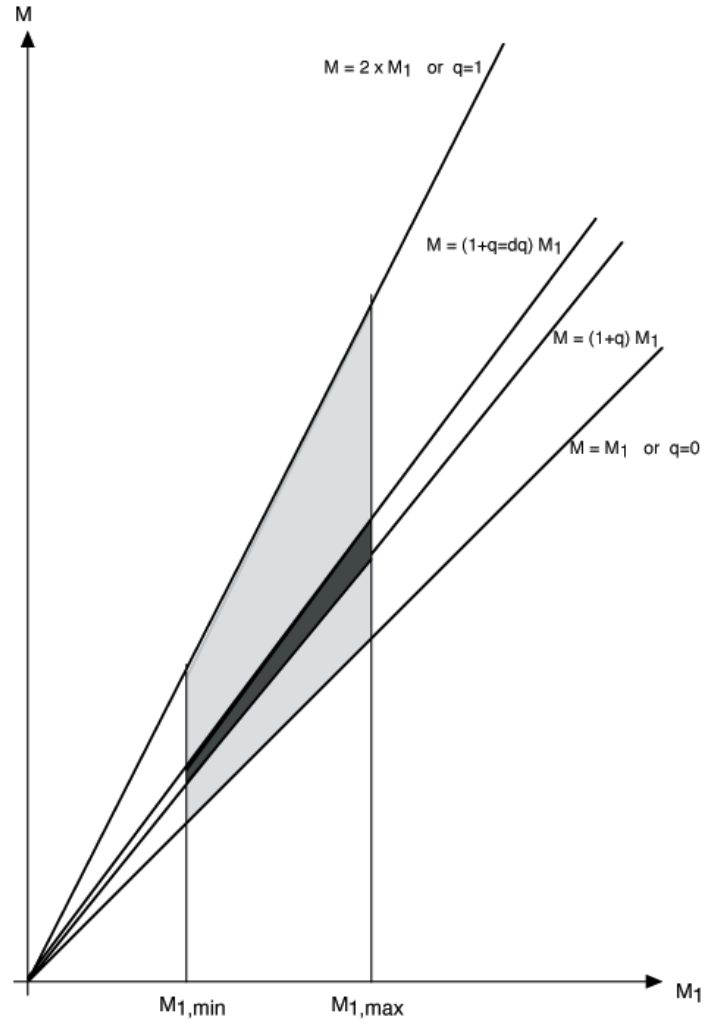

Fig. 2. A binary with primary mass $\in\left[M_{1, \min }-M_{1, \max }\right]$ is in the grey part of the $\left(M_{1}-M\right)$-plane. The black part contains those binaries $\in[q-q+\mathrm{d} q] . \mathrm{M} \in\left[M_{1}-2 \times M_{1}\right]$ is the total mass of the binary.

With the relations quoted between Eqs. (4) to (6) it is easy to show that the IMF of the total mass $\xi(M)$ of a binary obeys the same power law as the primaries of binaries:

$\xi(M)=\int_{M / 2}^{M} \xi\left(M, M_{1}\right)_{1} \mathrm{~d} M_{1} \propto M^{-\alpha}$.

The theoretical mass ratio distribution is found by determining the probability that a binary shows a mass ratio smaller than $q^{\star}: P\left(q<q^{\star}\right)$, by integrating the probability of occurrence below the straight line $M=\left(1+q^{\star}\right) M_{1}$ in Fig. 2 .

$$
\begin{aligned}
P\left(q<q^{\star}\right)= & \int_{M_{1, \min }}^{M_{1, \max }} \int_{M=M_{1}}^{M=\left(1+q^{\star}\right)} \xi\left(M, M_{1}\right) \xi\left(M_{1}\right) \mathrm{d} M \mathrm{~d} M_{1} \\
& \propto\left[\left(1+q^{\star}\right)^{1-\delta}-1\right] .
\end{aligned}
$$

The theoretical mass ratio distribution is the derivative of expression (8):

$\Psi(q) \propto(1+q)^{-\delta}$.

Expression (9) agrees with the distribution as derived by Kuiper (1935) $\left[\Psi(q) \propto(1+q)^{-2}\right]$ and needs no artificial constant $q_{o}$ in order to avoid a divergence for $q \rightarrow 0$ as was done by Hogeveen (1991) $\left[\Psi(q) \propto q^{-\alpha}\left(q \geq q_{o}\right) ; \Psi(q)=C\left(q \leq q_{o}\right)\right]$. The value of exponent $\delta$ in relation (9) will be determined by fitting a Monte Carlo simulation to the observed $q$-distributions of Table 2.

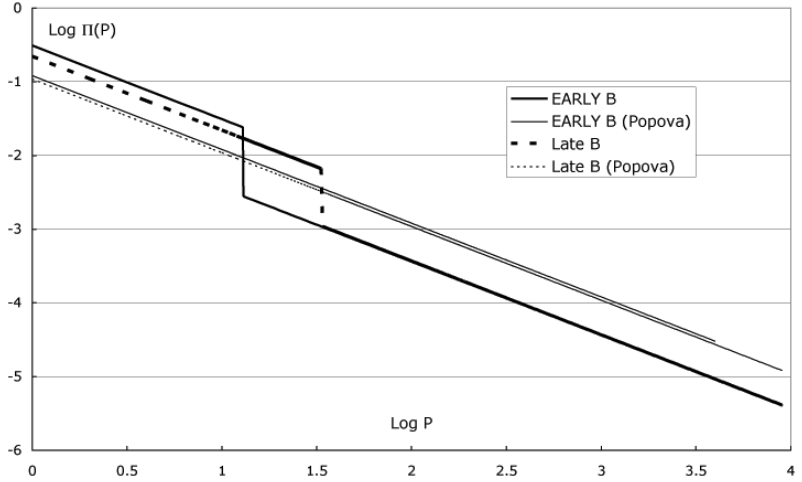

Fig. 3. The Popova distribution predicts too large orbital periods when it compares simulations with observations.

\subsection{Modeling the observed population of spectroscopic binaries}

\subsubsection{Modeling the observed distribution of periods}

First, we assume an initial distribution of orbital periods as given by Popova et al. (1982):

$\Pi(P)=\frac{A}{P}$ with $\int_{P_{\min }}^{P_{\max }} \Pi(P) \mathrm{d} P=1$.

If we try to fit this theoretical distribution to the observed periods of the non-evolved systems in the catalogue of Pourbaix (2005) we obtain a very poor fit as well for the binaries with an early B primary as for those with a late B primary component. The results of the Monte Carlo simulation (Sect. 2.4.3) depend moreover severely on the arbitrary choice of the limits of integration $P_{\min }$ and $P_{\max }$ in Eq. (10). The observed period distribution differs significantly from expression (10). The observed orbital periods of the non-evolved systems are on the other hand very well reproduced by an altered Popova distribution following different regimes at high and low orbital periods respectively. This new distribution is obtained by assigning a natural value to $P_{\max }$ linked to the largest observed orbital period. The long and short period regimes approximate the observed distribution best. The value of $P_{\min }$ follows from the normalisation of the distribution function. This procedure yields the following distribution with matches the observations almost perfectly:

- $79.3 \%$ of the observed systems with a late B primary follow a Popova distribution from $P_{\min }=0.93$ to $P_{\max }=33.37$; $20.7 \%$ follow a similar distribution between $P_{\min }=33.37$ and $P_{\max }=9000 \mathrm{~d}$;

- $79.1 \%$ of the observed systems with an early B primary follow a Popova distribution from $P_{\min }=1.02$ to $P_{\max }=$ $12.91 ; 20.9 \%$ follow a similar distribution between $P_{\min }=$ 12.91 and $P_{\max }=4000 \mathrm{~d}$.

The initial period distributions $\Pi(P)$ as derived from the observations are shown in Fig. 3. The distributions with two different regimes will be used in the subsequent analysis. 


\subsubsection{Simulating the population of binaries}

The observed mass ratio distribution $\Phi(q)$ for our sample of binaries with a non-evolved B type primary is constrained by Table 2. A Monte Carlo procedure with 100.000 binaries simulates a theoretical distribution $\Psi^{\prime}(q)$. This distribution is transformed into an observed distribution $\Phi^{\prime}(q)$ due to the model and the impact of observational selection effects. We propose an exponent $\delta$ in relation (9) fitting best the observed distribution $\Phi(q)$.

The Monte Carlo simulation uses the following distribution for a number of independent variables which are different for binaries with an early B primary and those with a late B primary at birth.

- The masses at birth of the initial primary follow a Salpeter-like distribution as given in relation (3) in which $\alpha$ will not differ drastically from the Salpeter-value $\alpha=2.35$. We have scanned $\alpha \in[0-5]$.

- The initial mass ratio distribution follows relation (9) in which the value of $\delta$ will be chosen so as to reproduce the data of Table 2 best.

- The distribution of the initial orbital periods follows the "broken" relation shown in Fig. 3: $\Pi(P)=\frac{C_{i}}{P}$, where the values of $C_{i}$ in the four different regimes (short period late $\mathrm{B}$, long period late $\mathrm{B}$, short period early $\mathrm{B}$, long period early B) obey the prescriptions mentioned in Sect. 2.4.1.

- The eccentricity follows a distribution as given by Hogeveen (1991): $\epsilon(\mathrm{e})=\frac{A}{e}$ with $\int_{e_{\min }=0.001}^{e_{\max }=0.7} \epsilon(\mathrm{e}) \mathrm{d} e=1$.

- The inclination angle follows the random distribution: $I(i)=A \times \sin i$ with $\int_{0}^{\pi} I(i) \mathrm{d} i=1$.

- Two assumptions have been tested for the spatial distribution of the binary systems:

the stars are uniformly distributed in a sphere: $D(r)=A \times$ $r^{2}$ with $\int_{r_{\min }}^{r_{\max }} D(r) \mathrm{d} r=1$;

the stars belong uniformly to a flat structure (e.g. Gould's belt): $D(r)=A \times r$ with $\int_{r_{\min }}^{r_{\max }} D(r) \mathrm{d} r=1$.

From these quantities a number of dependent variables can be calculated easily:

- the mass of the secondary $M_{2}=q \times M_{1}$;

- the absolute luminosities $\left(L / L_{\odot}\right)$ of the components are derived from their mass. This was done using Schaller et al. (1992) for stars with $M>0.8 M_{\odot}$. The middle of the main sequence was taken for this luminosity calibration. Allen (1973) was used for the lowest mass stars to which secondaries with a B type primary can belong. The absolute bolometric magnitude was then determined with the standard relation: $M_{\text {bol }}=4.75-2.5 \log \left(L / L_{\odot}\right)$;

- the absolute visual magnitude $M_{\mathrm{V}}$ was calculated from $M_{\text {bol }}$, using the bolometric corrections from Vanbeveren et al. (1998) for the high mass stars $\left(M>7 M_{\odot}\right)$ and Allen (1973) for the lower mass stars. The apparent visual magnitude is calculated from the standard relation $m_{V_{1,2}}=M_{\mathrm{V}_{1,2}}-5+5 \log r(\mathrm{pc})$

- the semi major axis of the system is given by the third Kepler law: $a\left(R_{\odot}\right)=4.2067\left[\left(M_{1}+M_{2}\right) P^{2}\right]^{1 / 3}$;

- the semi major axes of the components are found from the relations: $a=a_{1}+a_{2}$ and $\frac{a_{1}}{a_{2}}=\frac{M_{2}}{M_{1}}$;

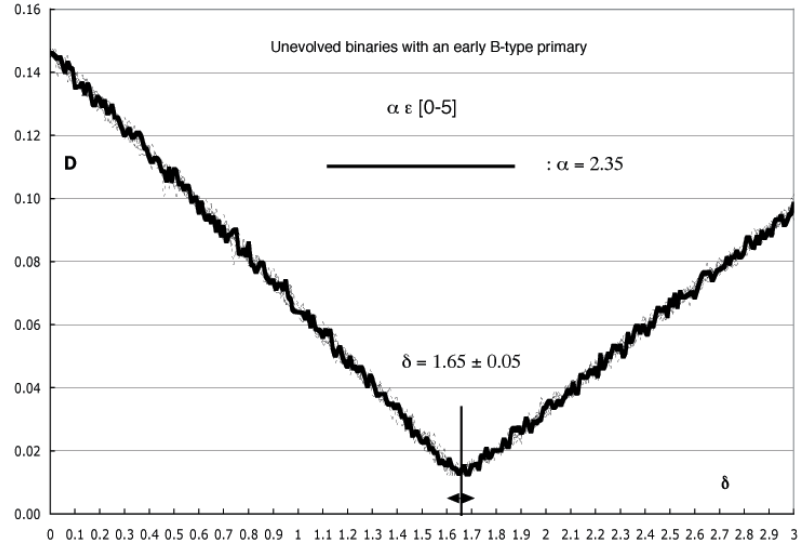

Fig. 4. Independent of the choice of $\alpha$ in the IMF in Eq. (3), binaries with an early B type primary tend to an initial mass ratio distribution $\Psi(q) \div(1+q)^{-\delta}$ with $\delta=1.65$ in the case that the mean observed mass ratio distribution is approximated by the model. The uncertainty introduced by a poor knowledge of $\alpha$ is at most 0.05 . For the late B type primaries a similar behaviour is found around $\delta=0.65$.

- this leads to the following radial velocity amplitude: $K_{1,2}\left[\mathrm{~km} \mathrm{~s}^{-1}\right]=\frac{50.6131 a_{1,2}\left(R_{\odot}\right) \sin i}{\left(1-e^{2}\right)^{1 / 2} P[\text { days }]}$.

\subsubsection{Modeling the selection effects}

A Monte Carlo simulation was elaborated separately for binaries with an early B type primary and those with a late B type primary. Each step produces 100.000 binaries with an unevolved B type primary. The primary masses follow an IMF-distribution $\xi\left(M_{1}\right)=C \times M_{1}^{-\alpha}$ (3) in which $\alpha$ can differ from the Salpeter-value $\alpha=2.35$, since primaries of binaries need not to follow the IMF of single stars. To each primary a secondary is attached through a $q$-distribution (9) with $\delta$ varying in the range $\in[-1 / 5]$. We have searched for the best the values of $\delta$ in the interval $\alpha \in[0-5]$ ranging from an unrealistic flat $(\alpha=0)$ to an unrealistic steep IMF $(\alpha=5)$. The value of $\delta$ that fits the observations best is barely dependent of the choice of $\alpha$, as can be seen in Fig. 4. An absolute visual magnitude is associated with both components by the massluminosity relation. The random distance generates apparent magnitudes for both components. A sufficiently small difference between these two magnitudes attaches a probabilty for the binary to be recognised as a SB2 as was done previously by Van Rensbergen (2001). The simulation produces further a random orbital period that follows the bimodal distribution of Fig. 3, a random eccentricity distribution as given by Hogeveen (1991) and a random distribution of the inclination angle. From these quantities, one determines subsequently $a, a_{1}$ and $K_{1}$. The minimum value of $K_{1}$ for a binary to be identified as a SB1 is determined from the $K_{1}$-values in the SB9-catalogue. A binary with $K_{1} \leq K_{1, \text { min }}$ will not be detected as SB1. When $K_{1} \geq$ $K_{1, \max }$ the system will be seen as a SB1 with equal probability as any binary with a larger value of $K_{1}$. The SB9 catalogue contains no B type stars with $m_{\text {vis }} \geq 11$ so that fainter ones have been removed from our simulations. We consider that stars with $m_{\text {vis }} \leq 9$ were detected with equal probability as stars looking 
Table 3. Parameters which are used in our simulation of 100.000 binaries. All values are best fits to the data of the SB9 catalogue.

\begin{tabular}{cccc}
\hline \hline Parameter & Unit & Early B primary & Late B primary \\
\hline$M_{1, \min }$ & $M_{\odot}$ & 7 & 2.5 \\
$M_{1, \max }$ & $M_{\odot}$ & 16.7 & 7 \\
$K_{1, \min }$ & $\mathrm{km} \mathrm{s}^{-1}$ & 2.8 & 2.3 \\
$K_{1, \max }$ & $\mathrm{km} \mathrm{s}^{-1}$ & 6 & 5 \\
$M_{2, \min }$ & $M_{\odot}$ & 0.08 & 0.08 \\
$M_{\max }$ & $M_{\odot}$ & $2 \times M_{1, \max }$ & $2 \times M_{1, \max }$ \\
$P_{\min }^{1}$ & days & 1.016 & 0.928 \\
$P_{\max }^{1}=P_{\min }^{2}$ & days & 12.912 & 33.370 \\
$P_{\max }^{2}$ & days & 4000 & 9000 \\
$e_{\min }$ & & 0.001 & 0.001 \\
$e_{\max }$ & & 0.7 & 0.7 \\
$d_{\min }$ & $\mathrm{pc}$ & 66 & 40 \\
$d_{\max }$ & $\mathrm{pc}$ & 1000 & 1000 \\
$m_{\mathrm{vis}, \min }$ & & 9 & 9 \\
$m_{\mathrm{vis}, \max }$ & & 11 & 11 \\
\hline & & &
\end{tabular}

apparently brighter. The SB9 catalogue is still incomplete, but we consider it as the most representative yet.

\subsection{Results}

In our search for the best values of $\delta$ we evaluated each model with a Kolmogorov-Smirnov test for which the maximum deviation $D$ was calculated in a column containing five $q$-bins. One can alter most of the numbers in Table 3 within reasonable ranges without harming the goodness of fit. The results yielded by a uniform distribution of the binaries in volume show no difference to those obtained assuming a uniform distribution in a plane.

The value of $\mathrm{D}$ is however extremely sensitive to variations of $\delta$ without being dependent of $\alpha$. Figure 4 shows the comparison between the "mean" value of the observations and our model in the case of binaries with an early B type primary. Each value of $\alpha$ in the IMF in relation (3) tends to the same value of $\delta \approx 1.65$ in relation (9). The maximum deviation $D$ is in this case very small $(D \approx 0.01)$. The best value of $\delta$ deviates much from the value found before ( $\delta \approx 3.37$, Van Rensbergen 2001) and the obtained maximum deviation is much smaller than in this former study ( $D \approx 0.01$ vs. $D \approx 0.05$ ), so that the fit with the observed mass ratios has been improved significantly. This is mainly due the use of the bimodal distribution of the initial orbital period shown in Fig. 3.

The agreement between our model and the observations is less striking for binaries with an unevolved late B type primary. Each value of $\alpha$ in the IMF in relation (3) tends to the same value of $\delta$ in relation $(9)(\delta \approx 0.65)$, with a maximum deviation $D \approx 0.045$. This value of $\delta$ deviates much from that found before $(\delta \approx 1.47)$ whereas the obtained maximum deviation is much smaller than in our former study $(D \approx 0.045$ vs. $D \approx$ 0.07), but still quite large.

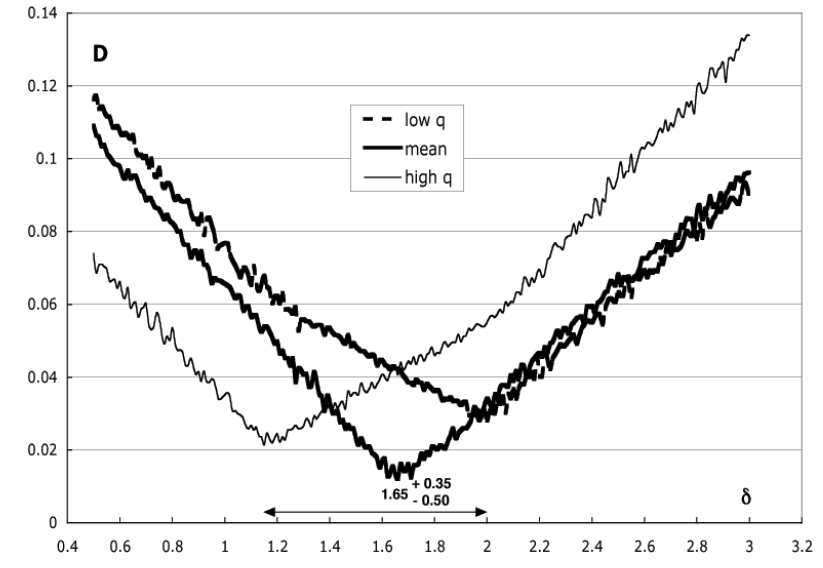

Fig. 5. An exact determination of the exponent $\delta$ is severely hindered observational uncertainties. This figure shows that the best value to explain the observed mass ratios of unevolved binaries with an early B type primary is $\delta=1.65$ if one uses the mean values of $K_{1}, K_{2}$ and $f(m)$ in SB9. If the uncertainties on these numbers all tend to lower mass ratios one obtains $\delta=2.0$, whereas $\delta=1.15$ is obtained with the assumption that all the uncertainties tend to higher mass ratios.

The uncertainty on $\delta$ is thus not dependent of change in the assumptions in Table 3 within reasonable limits, nor the choice of the exponent $\alpha$ in the IMF of the primaries, nor the assumptions about the uniform distribution in space of the binaries. The error on $\delta$ is mainly determined from the uncertainties in the catalogue which are emphasized in Table 2. Meeting the "low q" limit for binaries with an early B type primary one finds $\delta \approx 2.0$ with a maximum deviation $D \approx 0.03$. A flatter mass ratio distribution function fits the "high $q$ " limit with $\delta \approx 1.15$ with a maximum deviation $D \approx 0.02$. A mass ratio distribution with $\delta \approx 1.0$ and $D \approx 0.05$ represents best the "low $q$ " limit for binaries with a late B type primary, whereas the "high q" limit is represented best by an almost flat mass ratio distribution with $\delta \approx 0.1$ and $D \approx 0.06$. The fits achieved assuming extreme deviations from the mean values of $K_{1}, K_{2}$ and $f(m)$ are less good than those which are obtained using the mean values of $K_{1}, K_{2}$ and $f(m)$. The results are shown in Fig. 5.

As a safe conclusion we can state that:

- $\Psi(q) \propto(1+q)^{-\delta}$ with $\delta=0.65_{-0.55}^{+0.35}$ for unevolved binaries with a late B type primary.

- $\Psi(q) \propto(1+q)^{-\delta}$ with $\delta=1.65_{-0.50}^{+0.35}$ for unevolved binaries with an early B type primary.

The comparison of the models with the observations of Table 2 is shown in Fig. 6. The initial mass ratio distribution can be compared with earlier determinations:

- $\Psi(q) \propto(1+q)^{-2}$ : (Kuiper 1935).

- $\Psi(q)=C$ when $q \in[0-0.3] ; \Psi(q) \propto q^{-1.8}$ when $q \in[0.3-1]$ for all unevolved binaries with a B type primary (Hogeveen 1991).

The initial mass ratio distribution for binaries with an early B primary is steeper than for those with a late B primary. The agreement with the observations is however much better for the early B primaries so that a larger doubt remains on the obtained result about the late B primaries. 


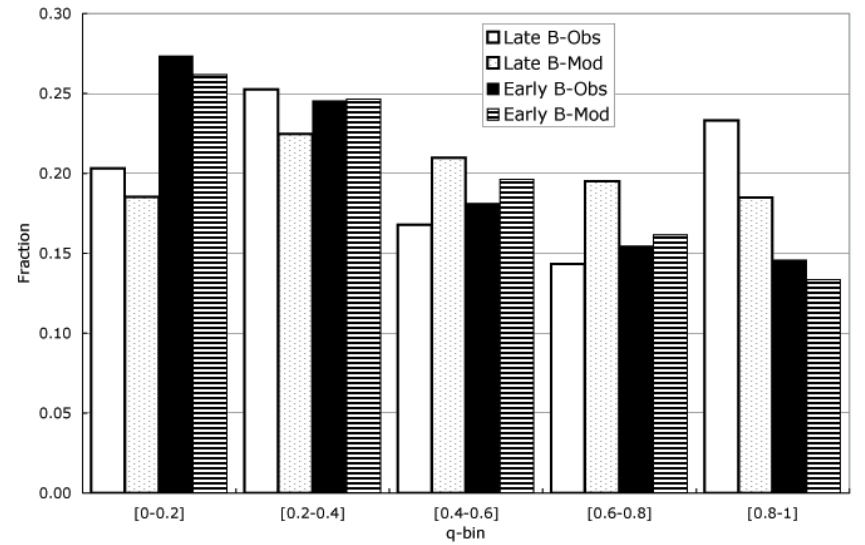

Fig. 6. Binaries with an unevolved late B primary following an initial $q$-distribution $\Psi(q) \div(1+q)^{-\delta}$ with $\delta=0.65$ match the mean values of the observations well, whereas those with an early B primary and $\delta=$ 1.65 show almost perfect agreement.

\section{Evolved binaries}

Starting from a population of unevolved binaries with a B type primary at birth as described in Sect. 2, we follow the evolution beyond the the RLOF-phase. Depending on the initial orbital period, binaries show Algol characteristics for a considerable amount of time during this phase. The phenomenon of loss of mass and angular momentum by the binary is a challenging question. The distribution of orbital periods and mass ratios of Algols calculated without mass and angular momentum loss is very different from that calculated with radically liberal scenarios. We will first compare the conservative simulation with the observations.

\subsection{Observed mass ratios and periods of Algols}

The distribution of the orbital period of Algols is well known and is shown in Fig. 7 for 378 Algols from Budding et al. (2004), respectively extended to 781 Algols with the catalogue of Brancewicz et al. (1996) and to 2545 including the semidetached Algols from Khopolov et al. (1998). These references mention the same periods for almost all systems which are common to the three catalogues and differ only in the number of Algols cited in them.

The distribution of mass ratios is however biased. From Budding et al. (2004) we selected Algols with a strongly established semi-detached status ( $\mathrm{sd} \geq 0.5$ ) for which we identified the mass ratio with $q_{\mathrm{MS}}$ so as to make the parameters of the most massive star fit Main Sequence (MS) correlations. The mass ratios $q_{\mathrm{LC}}$ obtained by the light curve solution and $q_{\mathrm{SD}}$ using the assumption of a semi-detached status yield however mass ratios that differ largely from $q_{\mathrm{MS}}$. Figure 8 shows that only the MS-assumption predicts a majority of Algols with a large mass ratio. Since Brancewicz et al. (1996) only restrain $q_{\mathrm{MS}}$, we will also use this value for our comparison between theory and observation. However, if $q_{\mathrm{LC}}$ or $q_{\mathrm{SD}}$ would be the rule one would expect a much smaller fraction of Algols with large mass ratios.

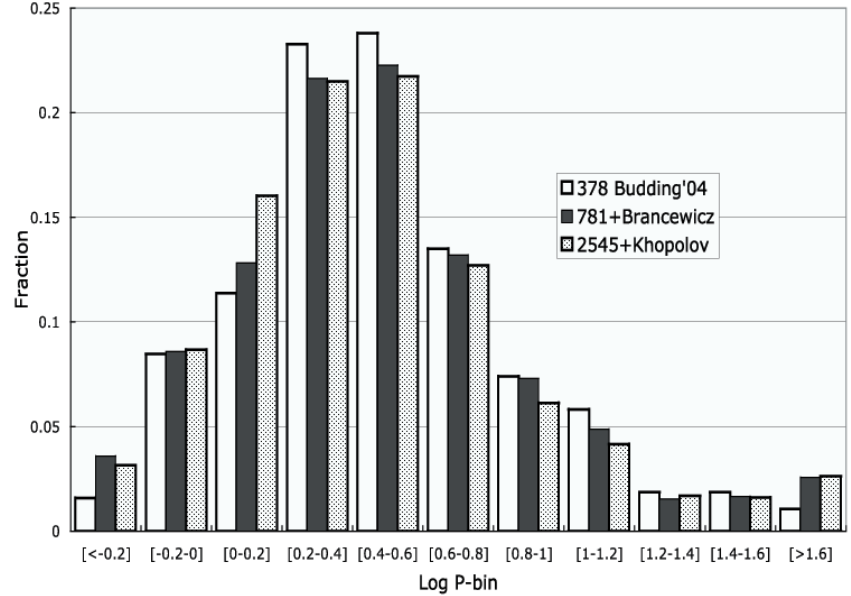

Fig. 7. The observed distribution of orbital periods of Algols.

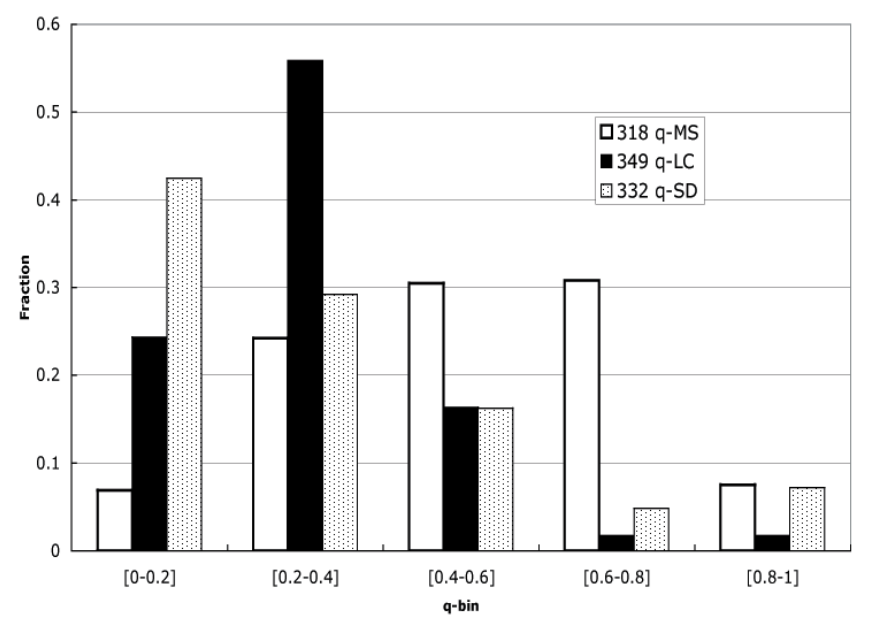

Fig. 8. The distribution of the mass ratio of Algols strongly depends on the method used to interpret the observations.

\subsection{Conservative simulation}

Our grid contains some 240 binaries with initial periods sufficiently small to lead to Case A RLOF (RLOF A): i.e. during $\mathrm{H}$ core burning of the donor. The donor is the initially primary star that becomes the less massive as soon as the Algol phase starts. From that moment on, one has that $M_{1}(t) \leq M_{2}(t)$ so that instead of the definition given in expression (1), the definition of the mass ratio becomes:

$q=\frac{M_{1}(t)}{M_{2}(t)}$

Evolutionary tracks are calculated for each system of our grid. A binary lives its era of "Algolism" (De Loore et al. 2004) when it matches the criterion of Peters (2001). All binaries show the Algol A aspect for some time during RLOF A. The drastical change of the mass ratio and the orbital period during this process can be followed in detail for each system in our grid. It happens frequently that RLOF $\mathrm{A}$ is followed by Case B RLOF (RLOF B): i.e. during $\mathrm{H}$ shell burning of the donor. Systems that have sufficiently large initial orbital periods so that RLOF A does not occur will also show a short-living Algol B appearance. These systems have been considered in 


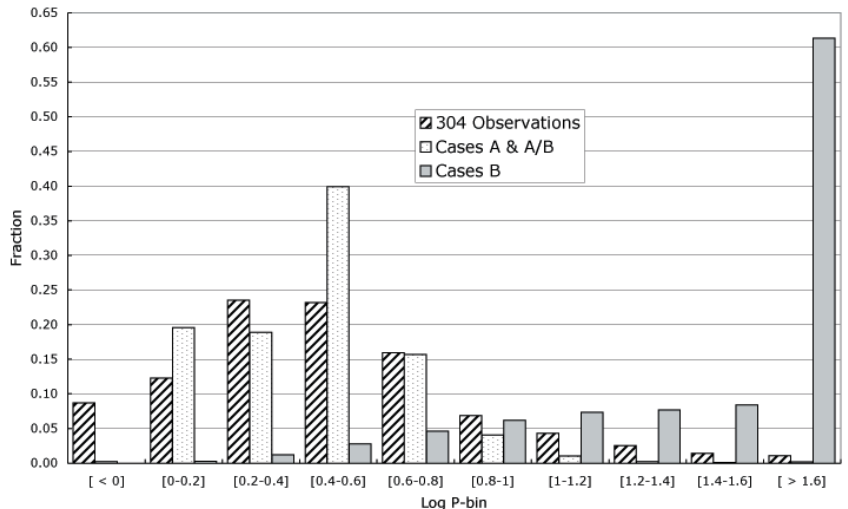

Fig. 9. Observed distribution of periods of Algols compared to conservative evolution for binaries with a B type primary at birth. Cases B produce an excess of long periods. Cases A follow the observed distribution better. There are far more Algol A than Algol B cases.

a previous paper (Van Rensbergen 2003) and are added to our simulation.

From our grid of conservative calculations we made a Monte Carlo simulation of the distribution of orbital periods and mass ratios of Algols. As starting conditions we selected:

- the primaries IMF of Salpeter (1955): $\zeta(M) \div M^{-2.35}$;

- an initial distrubution of orbital periods from Popova et al. (1982): $\Pi(P) \div \frac{1}{P}$

- an initial mass ratio distribution as derived in this paper. The distribution of the initial mass ratio $q=\frac{M_{2}^{0}}{M_{1}^{0}}$ obeys relation (9):

$\Psi(\mathrm{q}) \div(1+q)^{-\delta} ; \delta=1.65$ for early B and $\delta=0.65$ for late B primaries.

The catalogue of Budding et al. (2004) extended with the semi detached Algols from Brancewicz et al. (1996) supplies us with 422 Algols with a well defined value of $q_{\text {MS }}$. Figure 9 shows the observed distribution of orbital periods of 304 Algols which can be issued from conservative binary evolution with a B type primary at birth. We find more A than B cases, because the Algol A-phase goes on for a fraction of the nuclear time scale, whereas the Algol B phase lasts only for a fraction of the much shorter Kelvin-Helmholtz time scale. RLOF A produces Algols that match the observed distribution rather well. Since the Algol B cases peak towards the longest periods, a contribution of only a few $\%$ of B cases to the Algol population will mimic the observed period distribution well.

Figure 10 shows the observed mass ratio distribution of Algols that can be obtained from conservative binary evolution with a $\mathrm{B}$ type primary at birth. The mass ratio $q$ is herein as in expression (11) and as observers of Algols define this: $q=$ $\frac{M_{\text {donor }}}{M_{\text {gainer }}}=\frac{M_{1}(t)}{M_{2}(t)}$.

The mass ratios of the Algol B cases peak towards the smallest mass ratios, whereas RLOF A produces a majority of Algols in the $q$-bin [0.2-0.4]. Consequently an admixture of cases $\mathrm{A}$ and $\mathrm{B}$ will never reproduce the observed considerable number of Algols in the $q$-interval [0.4-1]. We may conclude that liberal binary evolution is required in order to describe the observed mass ratio distribution of Algols.

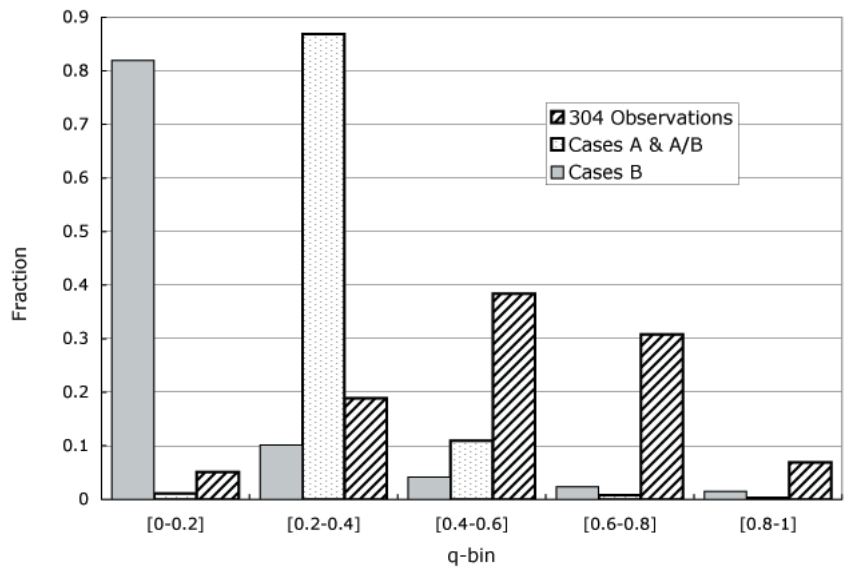

Fig. 10. Observed distribution of mass ratios of Algols compared to conservative evolution of binaries with a B type primary at birth. Cases B produce more than $80 \%$ Algols with $q \in[0-0.2]$. Cases A produce most of their Algols with $q \in[0.2-0.4]$. The fact that more than $70 \%$ Algols are located in $q \in[0.4-1]$ when one uses $q_{\text {MS }}$ as a standard excludes conservative evolution as the major channel through which Algols can be formed.

\subsection{Confining the liberal model}

Mass loss is defined by a parameter $\beta$ describing the fraction of the mass lost by the loser that is accreted by the gainer:

$\dot{M}_{\text {gainer }}=-\beta \dot{M}_{\text {donor }}$ with $0 \leq \beta \leq 1$.

Conservative evolution is described with $\beta=1$, whereas the liberal case uses values of $\beta<1$ that are not known beforehand. Conservative evolution implies that no angular momentum can be lost by the system, whereas the amount of loss of angular momentum in the liberal case is also a free parameter when no physical restrictions are implied. It is clear that realistic hydrodynamic calculations should show us the appropriate choice of the amounts of mass and angular momentum lost by a binary at any moment of the RLOF process. In the mean time we have performed a number of liberal evolutionary calculations and compared the results with the observations.

The time dependent parameter $\beta(\mathrm{t})$ should differ drastically from 1 - especially during the rapid phase of the RLOF process - since our calculations with $\beta$ close to one show hardly any difference with the conservative case.

For a given value of $\beta$, the amount of angular momentum lost by the system is defined by the locus where matter is removed from the system. Soberman et al. (1997) have argued that matter can be trapped in a Keplerian ring after transit across the second Lagrangian point $L_{2}$. The radius of the ring is $\eta$ times the semi major axis of the orbit. This Keplerian ring passes outside $L_{2}$ which is located at $\eta \approx 1.25$. This yields a minimum value of $\eta \approx 1.25$. Hydrodynamical calculations of Lubow \& Shu (1975) locate the ring at $\eta \approx 3$. This yields a maximum value of $\eta \approx 3$. A Keplerian ring located at $\eta \approx 2.25$ takes away the same amount of angular momentum as the corotating point $L_{2}$ would do. A value of $\eta \approx 2.25$ is thus a fair value in the interval [1.25-3] that can be used to calculate the change of the orbital period in this case. 


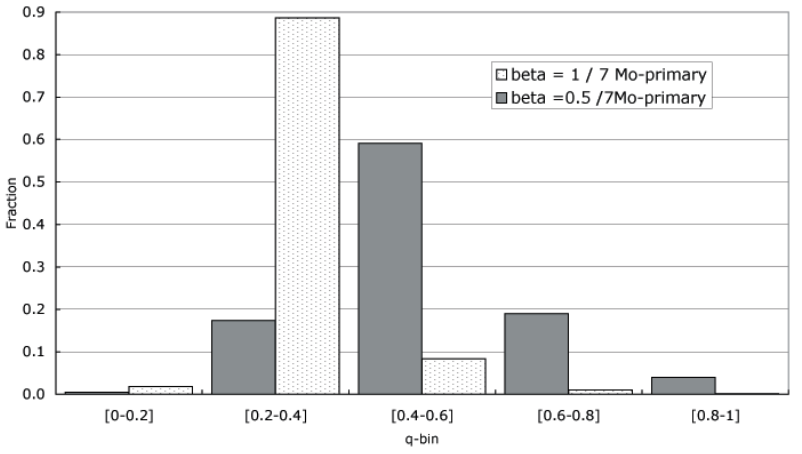

Fig. 11. Liberal evolution with a lot of mass loss $(\beta=0.5)$ and a little loss of angular momentum changes the mass ratio distribution of Algols drastically. The observed mass ratios in the $q$-interval [0.4-1] which were not produced by conservative evolution are created by this liberal model. This plot shows the result for Algols issued from RLOF A and a $7 M_{\odot}$ primary at birth.

Our calculations reveal that if the system loses a lot of matter and angular momentum across points located near $\eta \approx 2.25$ the orbital periods shrink drastically. Most binaries become mergers before they show Algolism. The obtained distribution of orbital periods of Algols is completely shifted towards the shortest periods. Since this conclusion conflicts with observations, the mass that leaves the system rather carries the angular momentum of points located nearer to $\eta \approx 0$ than to $\eta \approx 2.25$.

For all these reasons we performed a number of calculations with constant $\beta=0.5$ and angular momentum lost at the edge of the gainer. Until now only the representative cases with a $7 M_{\odot}$ primary at birth and initial periods leading to RLOF A have been performed. The assumption of mass loss through a point near $\eta=0$ does not alter the conservative distribution of the orbital periods of Algols. This agrees with the observations since the conservative orbital period distribution matches the observations fairly well. Liberalism however drastically alters the distribution of the mass ratios. Figure 11 shows that our liberal assumption $(\beta=0.5, \eta \approx 0)$ deviates radically from the conservative case. The $q$-bins [0.4-1] which were not populated in the conservative scenario are now populated properly as required by the observations.

\section{Conclusions}

1. The initial mass ratio distribution of binaries with an early B type primary is established in such a way that coincidence with observations is almost perfect. The distribution for binaries with a late B type primary fitting the observations best is flatter. However the agreement with the observations is in this case worse.

2. The uncertainties introduced by the error on the observations remain substantial.

3. Conservative RLOF produces Algols that have a distribution of the orbital periods that fits the observations well. However the conservative model produces smaller mass ratios than observed.
4. A liberal RLOF scenario with much mass-loss without much loss of angular momentum produces orbital periods and mass ratios of Algols that are in better agreement with observations.

5. The fraction of mass that needs to be lost by the binary strongly depends on the observed $q$-distribution that is assumed. If mass ratios of Algols are determined from MS correlations, the amount of mass lost by the binary will be larger than if one uses the LC or SD solutions.

6. Physical events that involve a liberal RLOF scenario at binaries with a B type primary at birth are yet not fully understood.

Acknowledgements. Part of this work was supported by the "Fonds voor Wetenschappelijk Onderzoek-Vlaanderen" project number G.0044.05.

We thank Peter Eggleton for his very valuable comments and suggestions.

\section{References}

Abt, H., \& Levy, S. 1985, ApJS, 59, 229

Allen, C. 1973, Astrophysical Quantities (London: The Athlone Press) Brancewicz, H., \& Dworak, T. 1980, Acta Astron, 30, 501; see also CDS, catalogue II/150 A, 1996

Budding, E., Erdem, A., Cicek, C., et al. 2004, A\&A, 217, 263

De Loore, C., \& Van Rensbergen, W. 2004, in Zdenek Kopal's Binary Star Legacy, ed. H. Drechsel, Space Sci. Rev., 296, 353

Eggleton, P. 2000, New Astron. Rev., 44, 111

Elmegreen, B. 1997, ApJ, 486, 944

Hogeveen, S. 1991, The mass ratio Distribution of Binary Stars, Ph.D. dissertation, University of Amsterdam

Khopolov, P., et al. 1998, CDS, catalogue II/214 A

Kuiper, G. 1935, PASP, 47, 15

Lubow, S., \& Shu, F. 1975, ApJ, 198, 383

Peters, G. 2001, Ap\&SS, 264, 79

Popova, E., Tutukov, A., \& Yungelson, L. 1982, Ap\&SS, 88, 55

Pourbaix, D., Tokovinin, A., Batten, A., et al. 2004, A\&A, 424, 727

Pourbaix, D. 2005, The 9th Catalogue of Spectroscopic Binary orbits, http://sb9.astro.ulb.ac.be/

Rana, N. 1987, ApJ, 184, 104

Refsdal, S., Roth, M., \& Weigert, A. 1974, A\&A, 36, 113

Salpeter, E. 1955, ApJ, 121, 161

Sarna, M. 1993, MNRAS, 262, 534

Schaller, G., Scharer, D., Meynet, G., \& Maeder, A. 1992, A\&AS, 96, 269

Soberman, G., Phinney, E., \& Van den Heuvel, E. 1997, A\&A, 327, 620

Vanbeveren, D. 1982, A\&A, 115, 65

Vanbeveren, D., Van Rensbergen, W., \& De Loore, C. 1998, The Brightest Binaries, Astrophys. Space Sci. Library, 232

Van Rensbergen, W. 2001, in The Influence of Binaries on Stellar population Studies, ed. D. Vanbeveren, Astrophys. Space Sci. Library, 264, 21

Van Rensbergen, W. 2003, in Stellar Astrophyics, ed. K. Cheng, K. Leung, \& T. Li, Astrophys. Space Sci. Library, 298, 117 